\title{
Valproic acid inhibits irradiation-induced epithelial-mesenchymal transition and stem cell-like characteristics in esophageal squamous cell carcinoma
}

\author{
AYAKO KANAMOTO $^{1}$, ITASU NINOMIYA ${ }^{1}$, SHINICHI HARADA ${ }^{2}$, TOMOYA TSUKADA ${ }^{1}$, \\ KOICHI OKAMOTO ${ }^{1}$, SHINICHI NAKANUMA ${ }^{1}$, SEISHO SAKAI ${ }^{1}$, ISAMU MAKINO ${ }^{1}$, \\ JUN KINOSHITA $^{1}$, HIRONORI HAYASHI ${ }^{1}$, KATSUNOBU OYAMA ${ }^{1}$, TOMOHARU MIYASHITA ${ }^{1}$, \\ HIDEHIRO TAJIMA $^{1}$, HIROYUKI TAKAMURA ${ }^{1}$, SACHIO FUSHIDA ${ }^{1}$ and TETSUO OHTA ${ }^{1}$ \\ ${ }^{1}$ Department of Gastroenterological Surgery, ${ }^{2}$ Center for Biomedical Research and Education, \\ Graduate School of Medical Science, Kanazawa University, Kanazawa, Ishikawa 920-8641, Japan
}

Received June 19, 2016; Accepted August 10, 2016

DOI: $10.3892 /$ ijo.2016.3712

\begin{abstract}
Esophageal carcinoma is one of the most aggressive malignancies, and is characterized by poor response to current therapy and a dismal survival rate. In this study we investigated whether irradiation induces epithelial-mesenchymal transition (EMT) in esophageal squamous cell carcinoma (ESCC) TE9 cells and whether the classic histone deacetylase (HDAC) inhibitor valproic acid (VPA) suppresses these changes. First, we showed that 2 Gy irradiation induced spindle cell-like morphologic changes, decreased expression of membranous E-cadherin, upregulated vimentin expression, and altered the localization of $\beta$-catenin from its usual membrane-bound location to cytoplasm in TE9 cells. Irradiation induced upregulation of transcription factors including Slug, Snail, and Twist, which regulate EMT. Stimulation by irradiation resulted in increased TGF- $\beta 1$ and HIF-1 $\alpha$ expression and induced Smad 2 and Smad3 phosphorylation. Furthermore, irradiation enhanced CD44 expression, indicating acquisition of cancer stem-like cell properties. In addition, irradiation enhanced invasion and migration ability with upregulation of matrix metalloproteinases. These findings indicate that single-dose irradiation can induce EMT in ESCC cells. Second, we found that treatment with $1 \mathrm{mM}$ VPA induced reversal of EMT caused by irradiation in TE9 cells, resulting in attenuated cell invasion
\end{abstract}

Correspondence to: Dr Itasu Ninomiya, Department of Gastroenterological Surgery, Graduate School of Medical Science, Kanazawa University, 13-1 Takara-Machi, Kanazawa, Ishikawa 920-8641, Japan E-mail: nino@staff.kanazawa-u.ac.jp

Abbreviations: ESCC, esophageal squamous cell carcinoma; EMT, epithelial mesenchymal transition; HDAC, histone deacetylase; DSB, double-strand break

Key words: esophageal squamous cell carcinoma, epithelial mesenchymal transition, histone deacetylase, stem cell, valproic acid and migration abilities. These results suggest that VPA might have clinical value to suppress irradiation-induced EMT. The reversal of EMT by HDAC inhibitors may be a new therapeutic strategy to improve the effectiveness of radiotherapy in ESCC by inhibiting the enhancement of invasion and metastasis.

\section{Introduction}

Esophageal carcinoma is one of the most common malignancies of the digestive system. In 2011, esophageal carcinoma was the fifth and eighth most common cause of death worldwide in men and women, respectively (1). Among this group of cancers, esophageal squamous cell carcinoma (ESCC) has high metastatic potential with frequent initial dissemination to regional lymph nodes and distant metastases at diagnosis, characteristics that underlie the majority of ESCC-related deaths $(2,3)$. Despite recent advances in clinical treatment, patients with ESCC exhibit a poor prognosis, with an average 5-year survival rate of $<20 \%$ worldwide due to distant metastases (4). Although the standard treatment for most solid cancers is surgical resection, most patients with unresectable malignant tumors, including ESCC, are treated by irradiation-based therapy. In recent years, $\sim 50 \%$ of all patients with solid cancer received radiation therapy. However, several recent preclinical and clinical studies have demonstrated that irradiation induces an increase in invasiveness and metastatic potential of several cancer cell types, including glioma, colon, breast, and lung cancer (5-7). Elucidation of the mechanisms underlying irradiation-induced metastasis is important to overcome failure of radiation therapy.

Epithelial mesenchymal transition (EMT) has become recognized as one of the major factors causing metastatic relapse and resistance to anticancer agents $(8,9)$. EMT is a crucial process in cancer progression, providing cancer cells with the ability to escape from the primary site, invade stromal tissues, and migrate to distant regions of the body. As a result of EMT epithelial cells lose their defined cell-cell/cell-substratum contacts and their structural/functional polarity and become spindle shaped and morphologically similar to activated fibro- 
blasts. At the molecular level, epithelial cells undergoing EMT are characterized by downregulation of epithelial makers (such as E-cadherin and $\beta$-catenin) and loss of cell polarity and intercellular adhesion molecules, concomitant with upregulation of mesenchymal markers (vimentin, N-cadherin, and fibronectin) and nuclear localization of $\beta$-catenin $(10,11)$. Cells that gain expression of mesenchymal markers show a frontrear polarity associated with weak cell-cell contact, increased motility, and resistance to apoptosis (10). Tumor cells that gain a more mesenchymal-like phenotype have migratory capacity at the expense of proliferative potential, and acquire resistance to DNA damage inducers including chemical agents and irradiation, thus becoming resistant to chemotherapy and radiotherapy $(8,9)$. Transforming growth factor- $\beta 1$ (TGF- $\beta 1$ ) is a multifunctional cytokine that modulates cell proliferation, differentiation, apoptosis, and extracellular matrix production (12). TGF- $\beta 1$ is known to play a critical role in EMT (13). TGF- $\beta 1$ mediates its effects through binding to a heteromeric complex of transmembrane serine/threonine kinases and a type II receptor, which results in activation of the receptor and initiation of Smad-dependent and -independent EMT pathways $(13,14)$. Environmental factors, including nicotine, ultraviolet light, and irradiation, also promote EMT $(15,16)$. Irradiation can cause many cellular effects, including apoptosis, senescence, and genomic instability, that may lead to cancer cell death. Conversely, irradiation also foments an increase in extra- and intracellular levels of TGF- $\beta 1$ in patients and induces acceleration of metastatic cancer progression $(6,17,18)$. Recently, irradiation was found to enhance cell migration, invasion, and metastasis through induction of EMT in various cancer cell lines, including ESCC cells (7,19).

Histone deacetylases (HDACs) are a family of enzymes that remove acetyl groups from histone and non-histone proteins and play an important role in regulating gene transcription and protein functions (20). HDAC inhibitors induce cell cycle arrest, differentiation, and apoptosis in vitro and in vivo (21-23). Over the last year several HDAC inhibitors have been introduced into clinical trials with successful results. Most epigenetic studies in the anticancer field have used valproic acid (VPA), the most potent HDAC inhibitor (24). The fact that VPA has been safely used in long-term therapy of patients with epilepsy over decades is a clear advantage, and phase I and II clinical trials of VPA in cancer have provided promising results $(25,26)$. In addition, tests of several protocols involving the use of VPA against diverse neoplasias are ongoing (20). VPA is a promising anticancer agent with effects correlated with the transcriptional regulation of specific cancer-related genes. We have noted the effectiveness of VPA as an anticancer agent and its ability to suppress collagen synthesis. In previous studies, we demonstrated that VPA enhances irradiation-induced cytotoxicity via chromatin decondensation and inhibition of DNA double-strand break (DSB) repair in human ESCC cells $(27,28)$. VPA also prevents the morphologic changes characteristic of activation and inhibits the expression of collagen type $1 \alpha 1$ and TGF- $\beta 1$ in human hepatic stellate cells (29). Recently, several reports have shown that HDAC inhibitors suppress metastatic potential in cancer cells by attenuating EMT $(30,31)$. However, there are no data on the potential role of VPA in the inhibition of irradiation-induced EMT.
The aim of this study was to evaluate the inhibitory effects of VPA on radiation-induced EMT in human ESCC cells and to reveal the underlying mechanisms.

\section{Materials and methods}

Cell lines, cell culture, and treatment. The TE9 cell line (human ESCC cell line, poorly differentiated) was kindly provided by Dr Tetsuro Nishihira (Kenotokorozawa Hospital, Saitama, Japan). Cells were grown in RPMI-1640 (Invitrogen, Tokyo, Japan) medium supplemented with $2 \mathrm{mM}$ glutamine, $10 \%$ fetal bovine serum (FBS; Nichirei Biosciences, Inc., Tokyo, Japan), $100 \mathrm{U} / 1$ penicillin, and $100 \mu \mathrm{g} / \mathrm{ml}$ streptomycin (Invitrogen) and maintained at $37^{\circ} \mathrm{C}$ in a $5 \% \mathrm{CO}_{2}$ incubator. The cells were seeded in gelatin-coated $75-\mathrm{cm}^{2}$ flasks (BioCoat, BD Biosciences, NJ, USA) and harvested with $0.25 \%$ trypsinEDTA before use.

Irradiation. Cultures were irradiated using MBR-150R-3 (Hitachi Medicotechnology, Hitachi, Japan) at a dose rate of $1.5 \mathrm{~Gy} / \mathrm{min}$. Power output of X-ray irradiation was $125 \mathrm{kV}$, $20 \mathrm{~mA}$. Forward-scattered radiation, 0.5-mm Al, and 0.2-mm $\mathrm{Cu}$ filters were used.

Reagents and antibodies. VPA was purchased from SigmaAldrich Co. (Tokyo, Japan) and used at concentrations of $0.1,0.5,1,5$ and $10 \mathrm{mM}$. VPA was dissolved in phosphatebuffered saline (PBS) to a stock concentration of $100 \mathrm{mM}$ and stored at $-20^{\circ} \mathrm{C}$. TGF- $\beta 1$ was purchased from Sigma-Aldrich and used at a concentration of $10 \mathrm{ng} / \mathrm{ml}$. Mouse monoclonal antibodies to E-cadherin, vimentin, TGF- $\beta 1$, Smad2, Smad3, matrix metalloproteinase 9 (MMP-9), HCAM (CD44), and $\beta$-catenin and rabbit polyclonal antibodies to phosphorylated Smad2 (p-Smad2), phosphorylated Smad3 (p-Smad3), Twist, Snail, Slug, and MMP-7 were obtained from Santa Cruz Biotechnology (Santa Cruz, CA, USA). Rabbit polyclonal antibodies to MMP-2 were obtained from Millipore (Billerica, MA, USA). Mouse monoclonal antibodies to $\beta$-actin and HIF-1 $\alpha$ were obtained from Sigma-Aldrich and Thermo Fisher Scientific (Rockford, IL, USA), respectively.

Cell viability assay. TE9 cells were plated in small dishes at a density of $5 \times 10^{4} / \mathrm{ml}$ in medium with $10 \%$ FBS and allowed to adhere for $24 \mathrm{~h}$ before incubation in serum-free medium for $24 \mathrm{~h}$. Cells were treated with vehicle or VPA $(0,0.1,0.5,1,5$, $10 \mathrm{mM}$ ) for $48 \mathrm{~h}$ and harvested by trypsinization. Cells were stained with a $0.4 \%$ trypan blue solution (Sigma Chemical Co., St. Louis, MO, USA), and counted on a Cellometer Vision automated cell counter (Nexcelom Bioscience, Lawrence, MA, USA) according to the manufacturer's protocol. Cell viabilities were represented as mean percentage relative to matched vehicle-treated cells for triplicate experiments with internal triplicates. At least three independent experiments were performed for statistical analysis.

Cell growth assay. The total number of living cells as a measure of proliferation was determined using a 3-(4,5-dimethylthiazol2-yl)-2,5-diphenyl tetrazolium bromide (MTT; Sigma-Aldrich) assay. Cells were seeded in quadruplicate in 95-well plates (BD BioCoat) with $5 \times 10^{3}$ cells per well and incubated for $24 \mathrm{~h}$ 
at $37^{\circ} \mathrm{C}$. After incubation, the supernatant was discarded and replaced with fresh serum-free medium. VPA was dissolved in PBS and added to the cell culture medium at various concentrations $(0,0.1,0.5,1,5$ and $10 \mathrm{mM})$. After 48 -h exposure to VPA, the cells were incubated at $37^{\circ} \mathrm{C}$ for $3 \mathrm{~h}$ with MTT solution $(500 \mu \mathrm{g} / \mathrm{ml})$ in fresh culture medium. The medium was removed and crystallized formazan dye in the cells was solubilized by addition of dimethylsulfoxide. The percentage inhibition was determined by comparing absorbance at $540 \mathrm{nM}$ of VPA-treated cells with that of untreated controls using a microplate reader (Bio-Rad 550; Bio-Rad, Japan). Three independent experiments were performed for statistical analysis.

Fluorescence immunocytochemistry. TE9 cells were grown on a 2-well Lab-Tek Chamber Slide System (Nalge Nunc International, Rochester, NY, USA) to $80 \%$ confluence and incubated for $24-48 \mathrm{~h}$ at $37^{\circ} \mathrm{C}$ in a humid atmosphere of $5 \% \mathrm{CO}_{2} / 95 \%$ air. Subconfluent cells were transferred to serum-free medium for $24 \mathrm{~h}$, and then treated with $2 \mathrm{~Gy}$ irradiation or $10 \mathrm{ng} / \mathrm{ml} \mathrm{TGF}-\beta 1$ with or without 3 -h pretreatment with $1 \mathrm{mM}$ VPA. At 24 - to $48 \mathrm{~h}$ after exposure to irradiation or TGF- $\beta 1$, morphologic changes were observed by phase-contrast microscopy. The cells were then subjected to immunostaining. Cells on coverslips were fixed with methanol and acetone $(1: 1 \mathrm{v} / \mathrm{v})$. After pretreatment with protein blocking serum for $10 \mathrm{~min}$ at room temperature to block non-specific binding, TE9 cells were incubated with primary antibody [anti-E-cadherin (1:200), anti-vimentin (1:200), or anti- $\beta$ catenin (1:100)] overnight at $4^{\circ} \mathrm{C}$. Following washes in PBS, cells were incubated with the appropriate Alexa Fluor ${ }^{\circledR} 488$ and $592 \mathrm{~nm}$ (Molecular Probes/Invitrogen, OR, USA; 1:400) for $1 \mathrm{~h}$ at room temperature. Nuclei were counterstained with bis-benzimide (Hoechst 33258; Sigma-Aldrich Co.) for 5 min and cells were visualized with an immunofluorescence microscope (BX50/BX-FLA; Olympus, Japan).

Invasion assay. Cell invasion ability was analyzed by an invasion assay using a BioCoat Matrigel Invasion Chamber for 24-well plates (BD Bioscience). In brief, Matrigels were rehydrated before use. Cells were treated with TGF- $\beta 1$ or irradiation as described above and seeded into the upper chamber system in serum-free media $\left(5 \times 10^{4}\right.$ cells/well) with the control or Matrigel membrane. The lower chamber contained $750 \mu \mathrm{l}$ of fresh medium with $10 \%$ FBS. After 24 -h incubation at $37^{\circ} \mathrm{C}$ in a humid atmosphere of $5 \% \mathrm{CO}_{2} / 95 \%$ air, the upper surface of the filter was wiped with a cotton swab and fixed in $100 \%$ methanol. Cells on the lower surface of the membrane were stained by hematoxylin and counted using a microscope. The number of cells in three random optical fields (x40 magnification) was averaged. Percentage invasion was calculated as the ratio of the number of invaded cells through the Matrigel membrane relative to that through the control membrane.

Migration assay. Cells were seeded in 6-well culture plates and grown to $\sim 80 \%$ confluence. The confluent cell monolayer was scraped with a $10-\mu l$ pipette tip. Cells were treated with TGF- $\beta 1$ or irradiation as described above. Images were captured immediately after wounding $(0 \mathrm{~h})$ and after $48-\mathrm{h}$ incubation at $37^{\circ} \mathrm{C}$ in a humid atmosphere of $5 \% \mathrm{CO}_{2} / 95 \%$ air. Percentage of migration was calculated as the ratio of migrated cells from the leading edge $48 \mathrm{~h}$ after scratching relative to that in control experiments.

Western blotting. Expression of EMT-related proteins was analyzed by western blotting. First, cells were lysed in RIPA buffer (Wako Pure Chemical Industries, Ltd., Osaka, Japan) containing a protease and phosphatase inhibitor cocktail (Sigma-Aldrich). The protein concentration of each sample was measured using a BCA protein assay kit (Thermo Scientific, Waltham, MA, USA). For sodium dodecyl sulfate-polyacrylamide gel electrophoresis (SDS-PAGE), $20 \mu \mathrm{g}$ of protein from each sample was separated on a $12.5 \%$ gradient polyacrylamide gel (e-PAGEL, ATTO Corp., Tokyo, Japan) and transferred to polyvinylidene difluoride membranes (Bio-Rad Laboratories). After incubation with blocking solution (EZ Block ATTO Corp.) at room temperature for $30 \mathrm{~min}$, the membranes were incubated with primary antibody overnight at $4^{\circ} \mathrm{C}$, followed by incubation with horseradish peroxidase-labeled anti-mouse or anti-rabbit IgG secondary antibody (Santa Cruz Biotechnology) for $1 \mathrm{~h}$ at room temperature. Immune complexes were detected using the ECL Plus Western blotting detection system (GE Healthcare UK, Ltd., Buckinghamshire, UK) and the Light Capture system (ATTO Co. Ltd.). The density of each band was quantified by the CS analyzer program (Atto Co. Ltd.). We used primary antibodies against the following proteins: E-cadherin (1:500), vimentin (1:500), $\beta$-catenin (1:1,000), TGF- $\beta 1$ (1:1,000), Smad2 (1:200), Smad3 (1:200), phosphorylated-Smad2 (p-Smad2) (1:200), p-Smad3 (1:200), Twist (1:200), Snail (1:200), Slug (1:200), pro-MMP-2 (1:200), pro-MMP-7 (1:200), pro-MMP-9 (1:200), and HIF-1 $\alpha$ (1:500). Antibody against $\beta$-actin $(1: 10,000)$ was used as an internal control.

Flow cytometry. To detect CD44, flow cytometry was performed using standard protocols. Cells were harvested with $0.25 \%$ trypsin-EDTA. Cell suspension and anti-CD44 antibody (1:20) were mixed and incubated for $15 \mathrm{~min}$ at room temperature. After centrifugation at $2,000 \mathrm{rpm}$ for $3 \mathrm{~min}$ at $4^{\circ} \mathrm{C}$ to remove debris, cells were analyzed on a BD FACS Calibur flow cytometer (Beckman Coulter, Brea, CA, USA).

Data analysis. All experiments were independently performed at least three times and the results were expressed as mean \pm SD. Data were analyzed with SPSS statistics 19 (SPSS Inc., Chicago, IL, USA) by Student's unpaired t-test and $\chi^{2}$ test. $\mathrm{P}<0.05$ was considered to indicate a statistically significant difference.

\section{Results}

In vitro effect of VPA on cytostasis and cytotoxicity of TE9 cells. Treatment of TE9 cells with increasing doses $(0,0.5,1$, 5 and $10 \mathrm{mM}$ ) of VPA for $48 \mathrm{~h}$ induced a significant dosedependent decrease in cell proliferation as assayed by MTT metabolism (Fig. 1A). Treatement with 5 and $10 \mathrm{mM} \mathrm{VPA}$ significantly inhibited TE9 cell proliferation to $55.2 \pm 5.3 \%$ $(\mathrm{P}=0.0045)$ and $52.5 \pm 5.0 \%(\mathrm{P}=0.004)$ compared with control, respectively. Trypan blue assays revealed no cytotoxic effect of VPA against TE9 cells even at the highest concentration (10 mM) (Fig. 1B). 
A

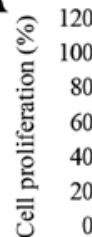

B

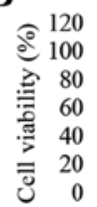

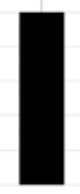

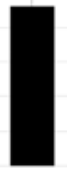

0.5

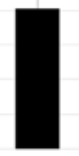

1

Concentration of VPA $(\mathrm{mM})$

0.1

0.5

1

Concentration of VPA (mM)

Figure 1. Effect of VPA on proliferation and viability of TE9 cells. (A) MTT assay, (B) trypan blue exclusion assay. Data are represented as percentage of control (mean \pm SEM) of three independent experiments $\left({ }^{*} \mathrm{P}<0.05\right)$. MTT, 3-(4,5-dimethylthiazol-2-yl)-2,5-diphenyltetrazolium bromide; SEM, standard error of the mean

The effect of VPA on morphologic changes induced by TGF- $\beta 1$ or irradiation in TE9 cells. Untreated TE9 cells showed a cobblestone-like morphology and strong cell-cell adhesion (Fig. 2A). Treatment with $10 \mathrm{ng} / \mathrm{ml}$ TGF- $\beta 1$ or 2 Gy of irradiation induced remarkable morphologic changes after $48 \mathrm{~h}$ of stimulation, including loss of cell polarity, appearance

of spindle-shaped cells, and enlargement of the cell space (Fig. 2B and C). However, TE9 cells that were pretreated with $1 \mathrm{mM}$ VPA did not show obvious morphologic changes (Fig. 2E and F).

The effect of VPA on changes in expression of epithelial and mesenchymal markers induced by TGF- $\beta 1$ or irradiation in TE9 cells. To investigate the inhibitory effect of VPA on EMT induced by TGF- $\beta 1$ or irradiation in TE9 cells, we examined the expression pattern of the epithelial marker E-cadherin and the mesenchymal marker vimentin with or without VPA treatment under TGF- $\beta 1$ or irradiation stimulation by immunocytochemistry and western blot analysis (Fig. 3). TE9 cells stimulated by TGF- $\beta 1$ or irradiation showed a significant decrease in E-cadherin expression and a concomitant increase in vimentin expression compared with control cells. Immunocytochemistry showed that E-cadherin expression at the membrane was completely lost after TGF- $\beta 1$ or irradiation stimulation. In contrast, cells that were pretreated with $1 \mathrm{mM}$ VPA showed less inhibition of E-cadherin expression than untreated cells when stimulated by TGF- $\beta 1$ or irradiation. Vimentin expression was strongly increased and uniformly distributed under TGF- $\beta 1$ or irradiation stimulation. Upregulation of vimentin expression by TGF- $\beta 1$ or irradiation stimulation was clearly inhibited by VPA.

Effect of VPA on $\beta$-catenin translocation promoted by TGF- $\beta 1$ or irradiation in TE9 cells. One characteristic of EMT is breakdown of the cytoplasmic cell adhesion complex. Breakdown of
A

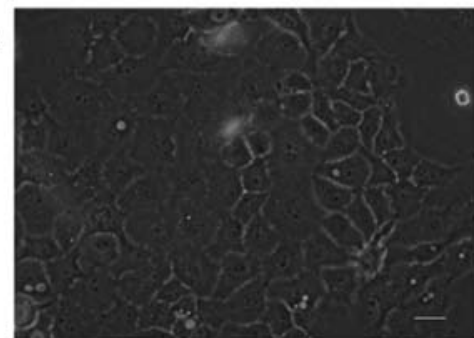

C

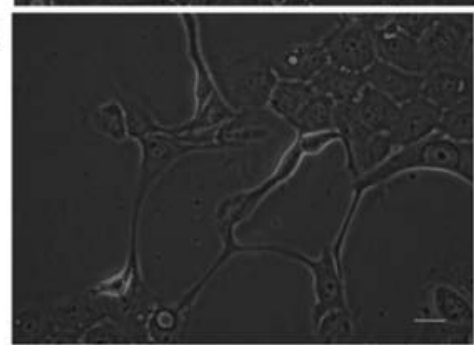

$\mathbf{E}$

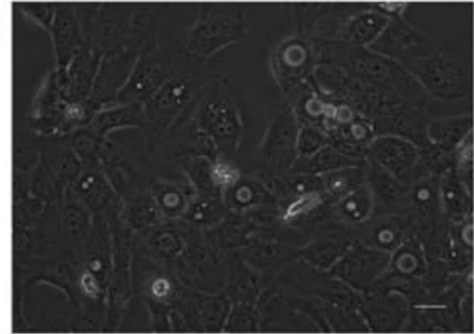

B

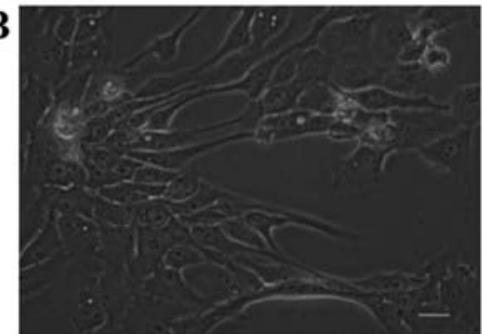

D
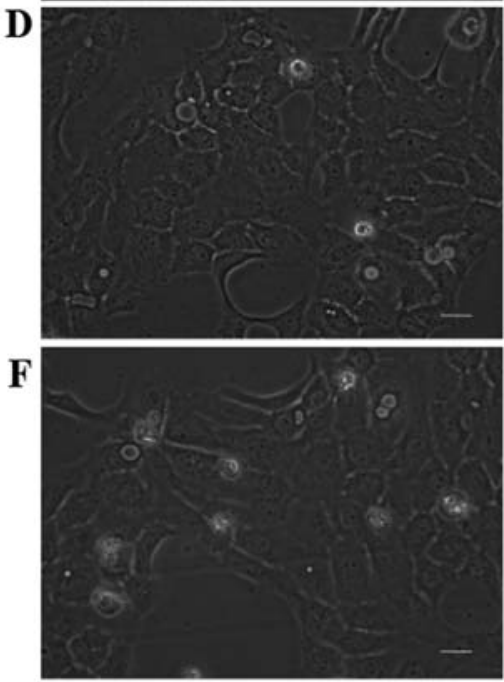

Figure 2. Effect of VPA on morphologic changes of TE9 cells induced by TGF- $\beta 1$ or irradiation. TE9 cells were incubated with $1 \mathrm{mM}$ VPA for $3 \mathrm{~h}$ followed by treatment with TGF- $\beta 1$ or irradiation. (A) Untreated control TE9 cells showed cobblestone-like morphology. (B and C) TGF- $\beta 1$ (10 ng/ml) or irradiation (2 Gy) induced morphologic transformation to a spindle shape in TE9 cells. (D) VPA (1 mM) had no effect on TE9 morphology compared with control. (E and F) VPA $(1 \mathrm{mM})$ inhibited spindle shape transformation induced by TGF- $\beta 1$ or irradiation. Scale bar, $10 \mu \mathrm{m}$. 
A
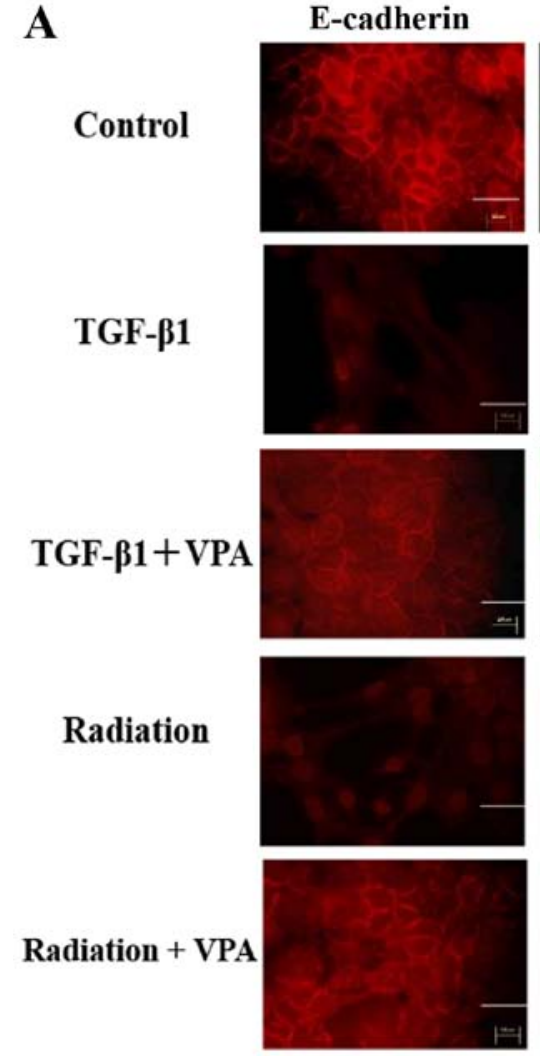
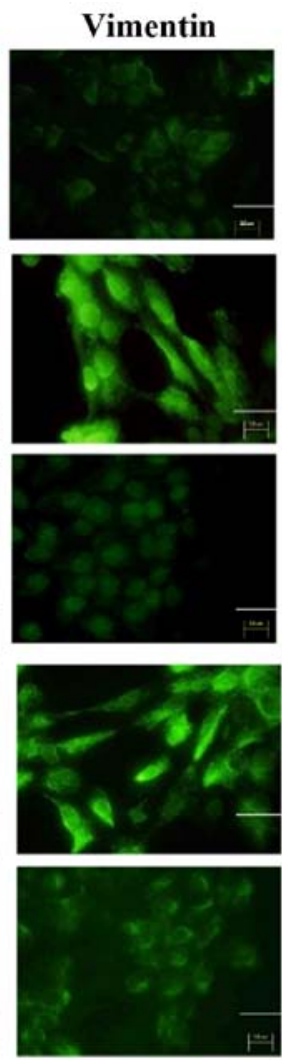
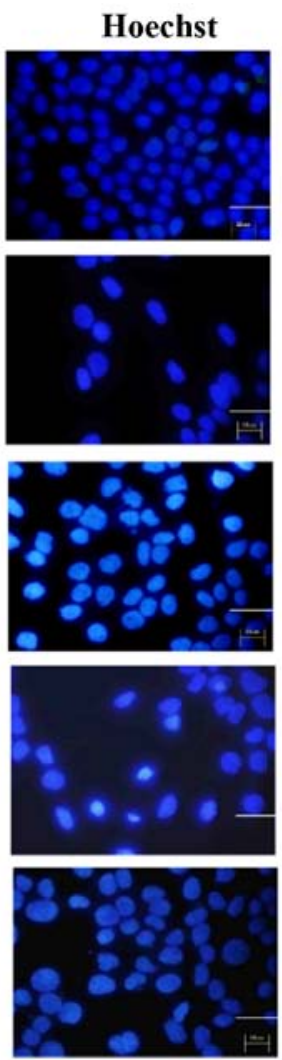
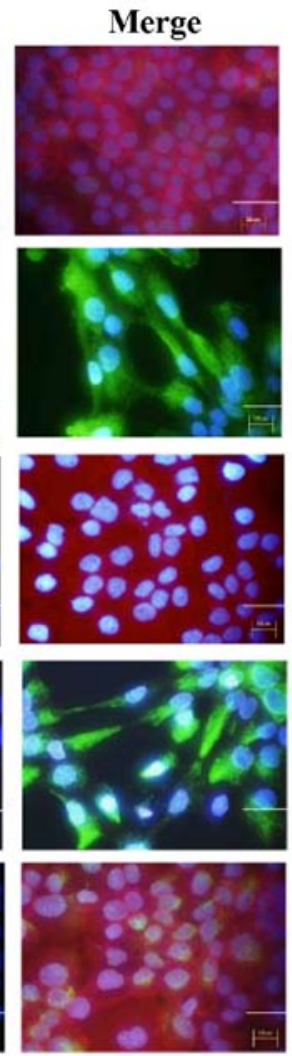

\section{B}

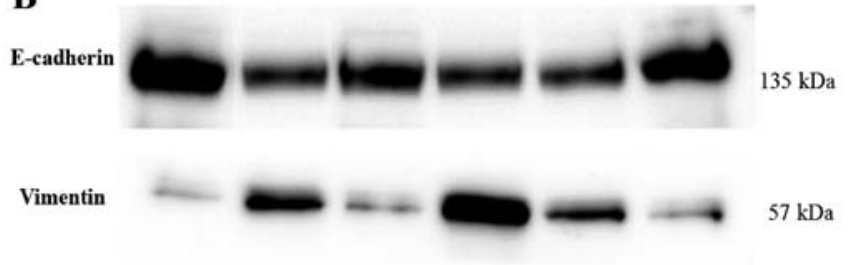

$\beta$-actin

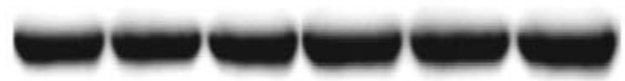

this complex causes a change in the localization of $\beta$-catenin from its usual membrane-bound location. Immunofluorescence studies showed that $\beta$-catenin translocated to the nucleus or cytoplasm from its usual membrane-bound location 24-48 h after treatment with TGF- $\beta 1$ or irradiation. Pretreatment with $1 \mathrm{mM}$ VPA inhibited these changes in the localization of $\beta$-catenin after TGF- $\beta 1$ or irradiation stimulation (Fig. 4A). In contrast, the total amount of $\beta$-catenin protein assessed by western blot analysis was not altered by stimulation with TGF- $\beta 1$ or irradiation or by pretreatment with VPA (Fig. 4B).

Effect of VPA on invasion and migration promoted by TGF- $\beta 1$ or irradiation stimulation in TE9 cells. To further understand the action of VPA on EMT, we investigated the effect of VPA on the enhancement of the invasive and migratory activity of TE9 cells by TGF- $\beta 1$ or irradiation. Cells treated with TGF- $\beta 1$ or irradiation showed 1.55 -fold $(\mathrm{P}=0.041)$ and 1.69 -fold
Figure 3. Effect of VPA on changes in the expression of epithelial and mesenchymal markers induced by TGF- $\beta 1$ or irradiation in TE9 cells. Expression pattern of epithelial marker E-cadherin and mesenchymal marker vimentin with or without VPA $(1 \mathrm{mM})$ under TGF- $\beta 1(10 \mathrm{ng} / \mathrm{ml})$ or irradiation (2 Gy) stimulation was examined by fluorescent immunocytochemistry (A) and western blot analysis (B). TE9 cells were stimulated by TGF- $\beta 1(10 \mathrm{ng} / \mathrm{ml})$ or irradiation $(2 \mathrm{~Gy})$ with or without VPA $(1 \mathrm{mM})$. Nuclei were stained by Hoechst 33258. Scale bar, $25 \mu \mathrm{m}$.
$(\mathrm{P}=0.038)$ enhanced invasion ability respectively compared with the control. Pretreatment with $1 \mathrm{mM}$ VPA decreased the enhancement of TE9 cell invasiveness by TGF- $\beta 1$ or irradiation (0.65-fold, $\mathrm{P}<0.01$ and 0.63 -fold enhancement, $\mathrm{P}<0.05$, respectively) (Fig. 5A and $\mathrm{B}$ ).

In the migration assay, TGF- $\beta 1$ - or irradiation-treated TE9 cells migrated almost completely across the leading edge after 48 h. TGF- $\beta 1$ or irradiation significantly increased TE9 cell migration ability (1.49-fold, $\mathrm{P}<0.001$ and 1.31 -fold, $\mathrm{P}=0.03$, respectively). Treatment with $1 \mathrm{mM}$ VPA significantly suppressed the enhancement of TE9 cell migration ability by TGF- $\beta 1$ or irradiation stimulation $(0.82$-fold, $\mathrm{P}<0.01$ and 0.86-fold, $\mathrm{P}<0.05$, respectively) (Fig. $5 \mathrm{C}$ and $\mathrm{D}$ ).

Effect of VPA on the expression of EMT-related proteins and MMPs in TE9 cells. Interaction of TGF- $\beta 1$ and TGF- $\beta$ receptor type I (T $\beta R-I)$ leads to phosphorylation of Smad2 
A

control

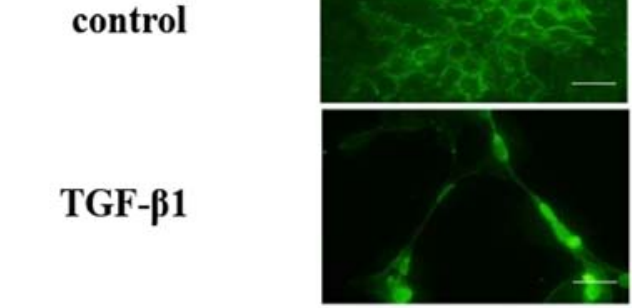

TGF- $\beta 1+$ VPA

Radiation

Radiation + VPA
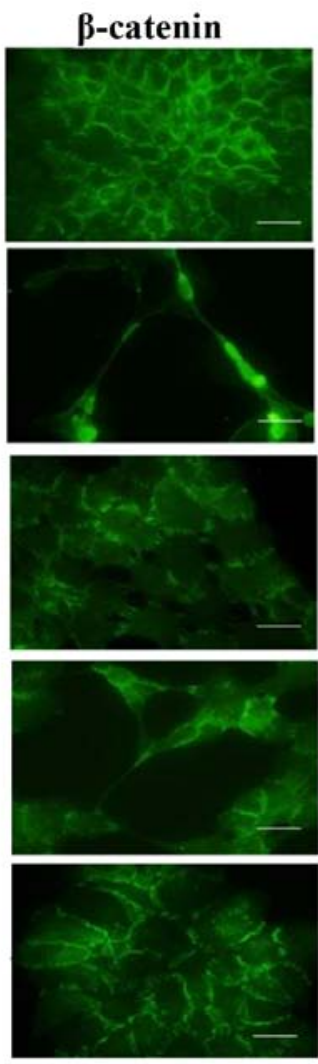

Hoechst
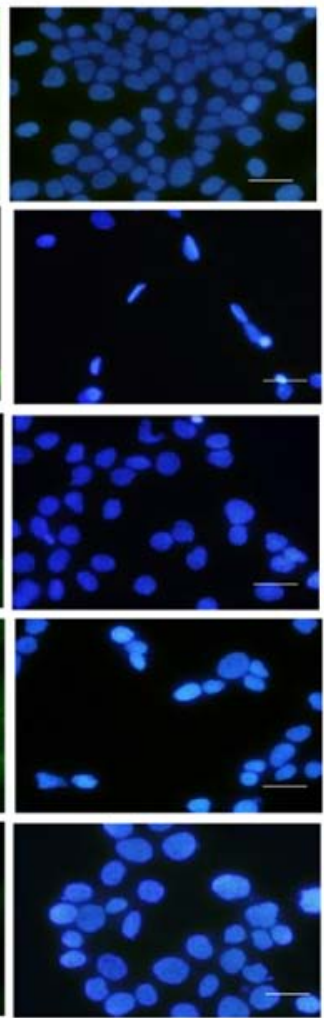
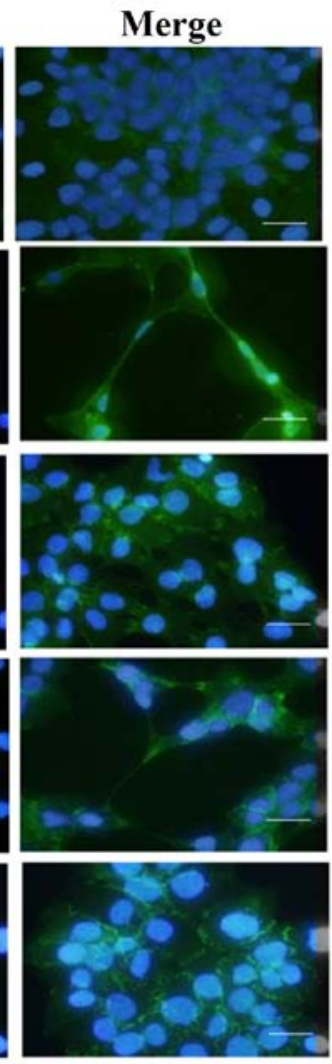

\section{B}

$\beta$-catenin

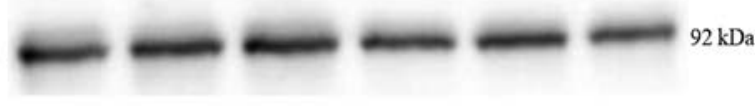

$\beta$-actin

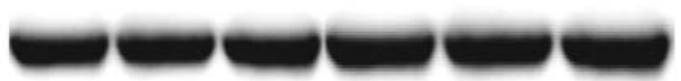

TGF- $\beta 1$

Radiation

VPA

and Smad3, the key mediators of TGF- $\beta 1$ signaling. Therefore, we examined TGF- $\beta 1$ expression and Smad 2 and Smad3 phosphorylation after stimulation by TGF- $\beta 1$ or irradiation. Stimulation by TGF- $\beta 1$ or irradiation resulted in increased TGF- $\beta 1$ expression. Consistent with this finding, stimulation by TGF- $\beta 1$ or irradiation also induced Smad 2 and Smad3 phosphorylation. Administration of VPA (1 mM) abolished both responses. Total Smad2 and Smad3 were abundant in control cells, and expression levels were not affected by TGF- $\beta 1$, irradiation, or VPA treatment (Fig. 6). Exposure of serum-starved TE9 cells to TGF- $\beta 1$ or irradiation resulted in increased phosphorylation of Smad2 and Smad3. Incubation with $1 \mathrm{mM}$ VPA suppressed phosphorylation of Smad2 and Smad 3 after TGF- $\beta 1$ or irradiation stimulation, whereas expression of total Smad2 and Smad3 was not affected (Fig. 6A), confirming that VPA can block TGF- $\beta 1$ signaling. We further delineated the link between TGF- $\beta 1$ - or irradiation-induced
Figure 4. Effect of VPA on $\beta$-catenin translocation promoted by TGF- $\beta 1$ or irradiation in TE9 cells. Localization and amount of $\beta$-catenin with or without VPA $(1 \mathrm{mM})$ under TGF- $\beta 1(10 \mathrm{ng} / \mathrm{ml})$ or irradiation stimulation was analyzed by fluorescent immunocytochemistry (A) and western blot analysis (B). TGF- $\beta 1(10 \mathrm{ng} / \mathrm{ml})$ or irradiation (2 Gy) treated cells showed changes in localization of $\beta$-catenin from their usual cell membraneassociated site to nuclear or cytoplasmic localization. Pretreatment of cells with $1 \mathrm{mM}$ of VPA prevented the observed changes in localization of $\beta$-catenin. The total protein level of $\beta$-catenin was not altered. Scale bar, $25 \mu \mathrm{m}$.
HIF-1 $\alpha$ expression and EMT progression by showing that VPA $(1 \mathrm{mM})$ suppressed HIF-1 $\alpha$ expression. In addition, TGF- $\beta 1$ or irradiation strongly induced expression of the gelatinases MMP-2, MMP-7, and MMP-9 whereas VPA strongly inhibited the induction of these enzymes (Fig. 6B).

Effect of VPA on induction of transcription factor expression by TGF- $\beta 1$ or irradiation stimulation in TE9 cells. Several key inducers of EMT are transcription factors such as Twist, Snail, and Slug that repress E-cadherin expression. Western blot analysis showed that TGF- $\beta 1$ or irradiation stimulation induced upregulation of Twist, Snail, and Slug expression (Fig. 7). Pretreatment with VPA resulted in inhibition of Twist, Snail, and Slug expression.

Effect of VPA on the induction of stem cell markers by TGF- $\beta 1$ or irradiation stimulation in TE9 cell. By western blotting, 
A
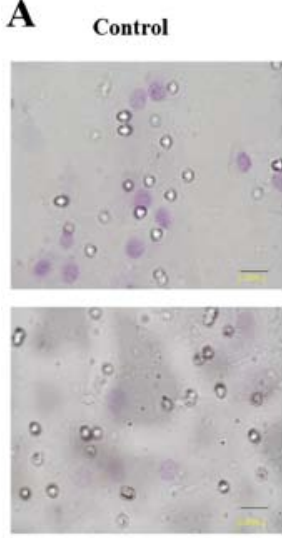

VPA

C

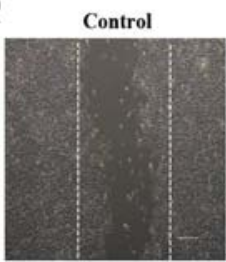

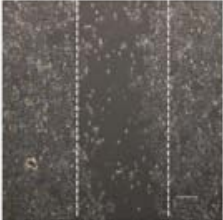

VPA

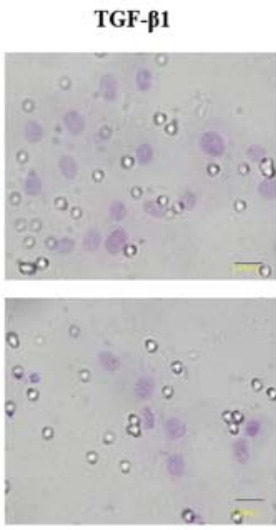

TGF- $\beta 1+V P A$

TGF- $\beta 1$
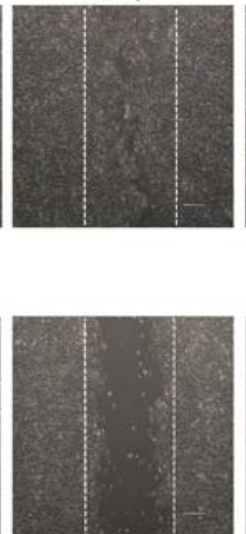

TGF- $1+$ + VPA
Radiation
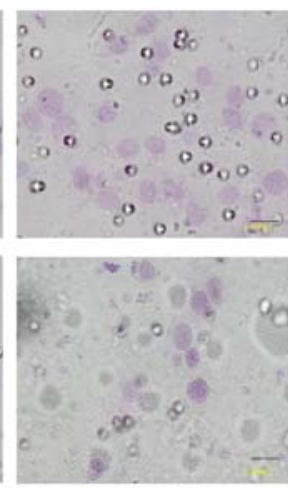

Radiation+VPA
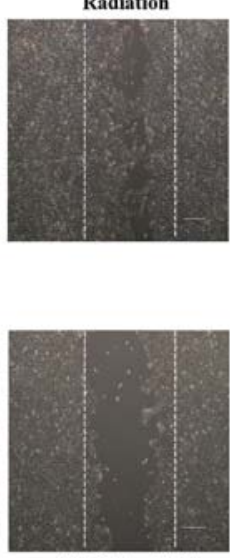

Radiation+VPA

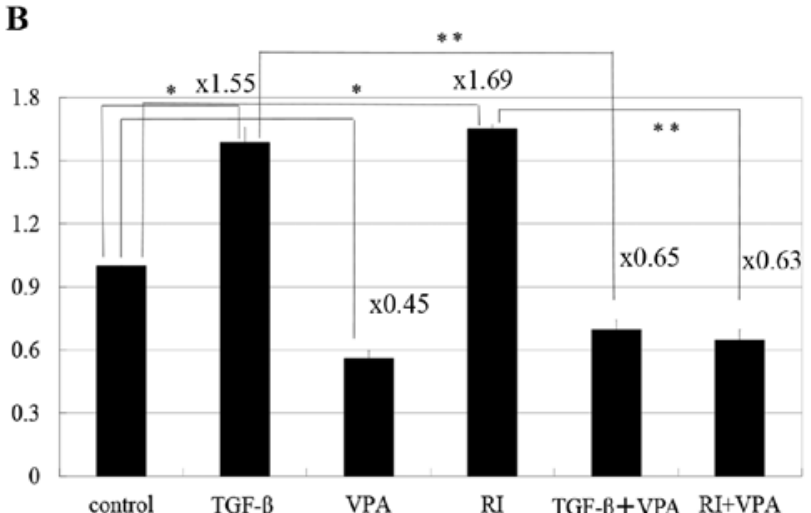

* $\mathrm{P}<0.05$

Figure 5. Effect of VPA on invasion and migration promoted by TGF- $\beta 1$ or irradiation stimulation in TE9 cells. Effect of VPA (1 mM) on invasion and migration promoted by TGF- $\beta 1(10 \mathrm{ng} / \mathrm{ml})$ or irradiation ( $2 \mathrm{~Gy})$ stimulation in TE9 cells was analyzed by invasion assay (A and B) and migration assay (C and D). (A) Representative photomicrograph of invaded TE9 cells. (B) Invaded cells in each group was counted and compared. (C) Representative photomicrograph of scratch migration asssay. (D) Migrated cells in each group was counted and compared. TGF- $\beta 1$ (10 ng/ml) or irradiation ( 2 Gy) significantly stimulated the invasion and migration ability of TE9 cells. VPA $(1 \mathrm{mM})$ significantly inhibited the invasion and migration ability induced by TGF- $\beta 1$ ( $10 \mathrm{ng} / \mathrm{ml})$ or irradiation (2 Gy) stimulation. ${ }^{*} \mathrm{P}<0.05,{ }^{* *} \mathrm{P}<0.001$. RI, radiation. Scale bar (A and B), $10 \mu \mathrm{m}$; (C and D), $100 \mu \mathrm{m}$.

A

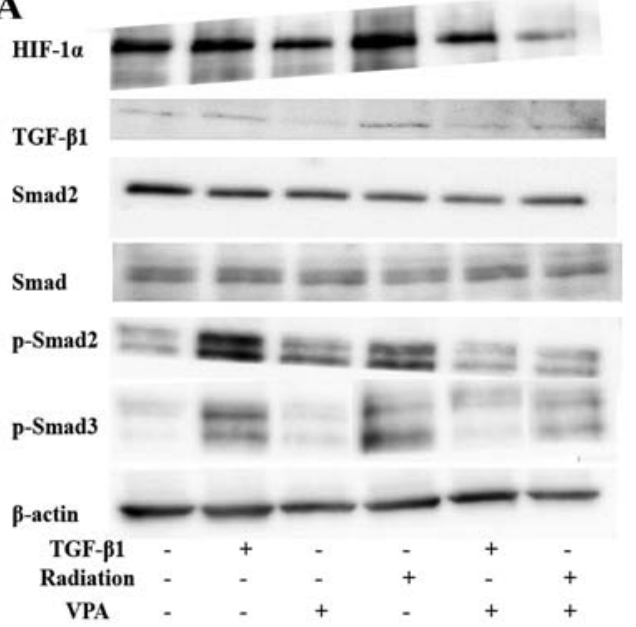

$132 \mathrm{kDa} \quad \mathrm{B}$

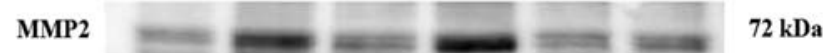

$12.5 \mathrm{kDa}$

55-60 kDa

MMP7

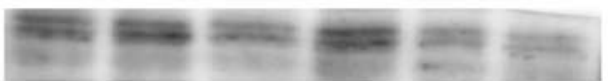

$30 \mathrm{kDa}$

$55-60 \mathrm{kDa}$

MMP9

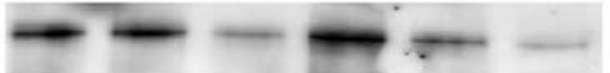

92 kDa

55-60 kDa

55-60 kDa

$\beta$-actin

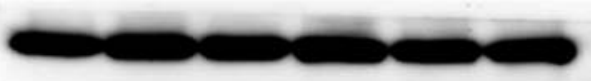

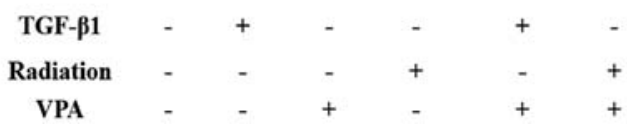

Figure 6. Effect of VPA on the expression of EMT-related protein and MMPs in TE9 cells. Effect of VPA (1 mM) on the expression of EMT-related protein (TGF- $\beta 1$, smad2, smad3, p-smad2, p-smad3 and HIF-1 $\alpha$ ) (A) and MMPs (B) was analyzed by western blot analysis.

the level of total CD44 protein was increased by TGF- $\beta 1$ treatment or irradiation and was decreased by VPA (Fig. 8A).
Fluorescence activated cell sorting (FACS) analysis also showed an increase in CD44 levels in TGF- $\beta 1$ or irradiation- 
Twist

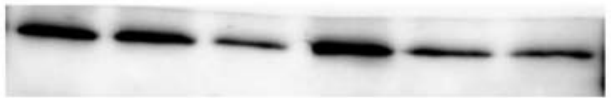

$28 \mathrm{kDa}$

Snail

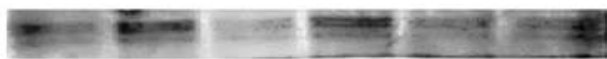

$29 \mathrm{kDa}$

Slug

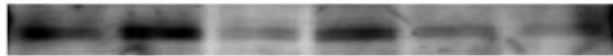

$30 \mathrm{kDa}$

\section{$\beta$-actin}

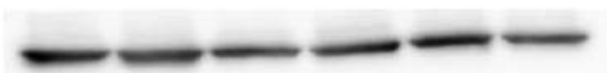

TGF-B1

Radiation

.

VPA

Figure 7. Interactive effect of VPA on transcription factors induced by TGF- $\beta 1$ or irradiation stimulation. Effect of VPA $(1 \mathrm{mM})$ on the expression of transcription factors (twist, snail and slug) promoted by TGF- $\beta 1$ (10 ng/ $\mathrm{ml}$ ) or irradiation (2 Gy) stimulation in TE9 cells was analyzed by western blot analysis.

A

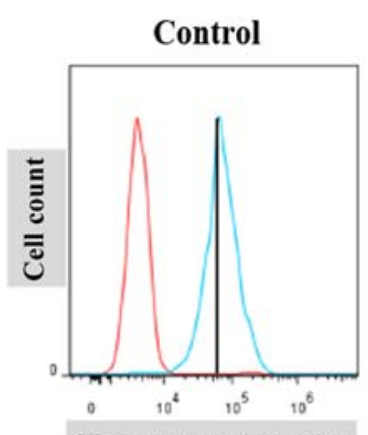

Fluorescence intensity

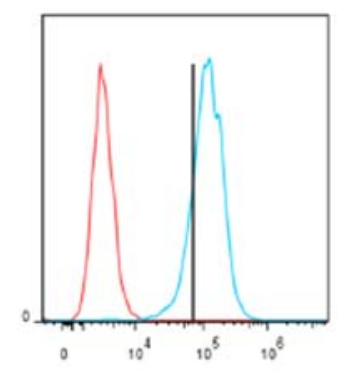

B
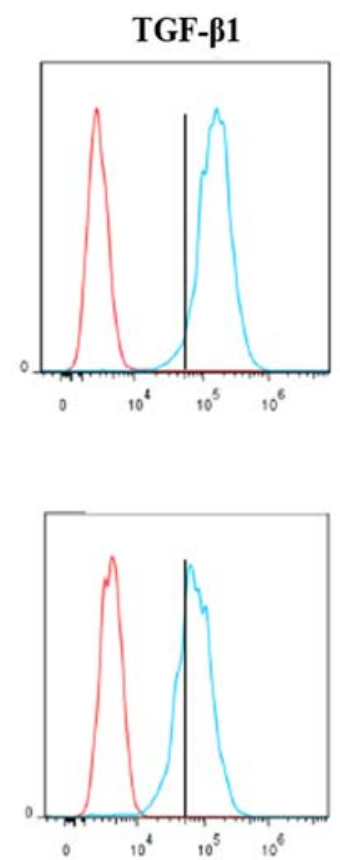

TGF- $\beta 1+$ VPA treated TE9 cells that was inhibited by VPA (Fig. 8B). These results indicate that TGF- $\beta 1$ or irradiation exposure can alter cellular expression of markers associated with cancer stemlike cell properties and that VPA inhibits transformation to a cancer stem-like phenotype.

\section{Discussion}

In this study, we demonstrated that irradiation induced morphologic and molecular alternations consistent with acquisition of a mesenchymal-like phenotype in ESCC cells. As a result of induction of EMT, ESCC cells attained invasive and migratory potential. In addition, the HDAC inhibitor VPA inhibited growth, invasion, migration, and the transformation to cancer stem-like phenotype associated with TGF- $\beta 1$ or irradiation stimulation of ESCC cells.

Radiotherapy plays an important clinical role as the major non-surgical treatment for esophageal cancer. However, previous studies have reported that irradiation may enhance the metastatic potential of residual cancer $(6,18)$. Local failure and distant metastasis are the primary causes of radiotherapy

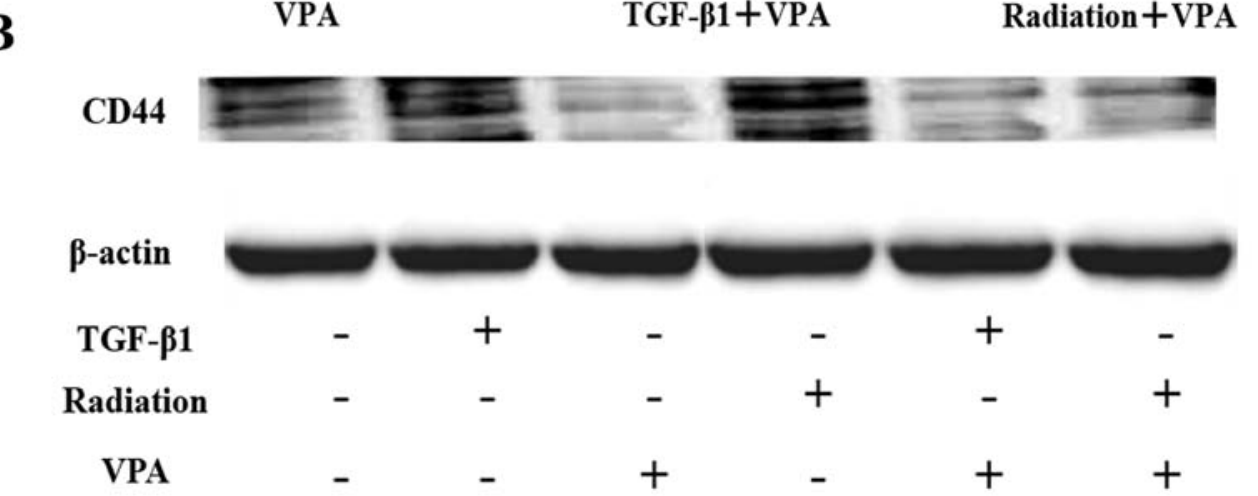

Figure 8. Effect of VPA on the expression of CD44 in TE9 cells. The effect of VPA (1 mM) on the expression of CD44 was analyzed by western blot analysis (A) and flow cytometry (B). 
failure. Therefore, tumor recurrence and metastasis might be associated with tumor biological behavior, as well as with EMT induced by irradiation. Recently, examination of a radioresistant ESCC cell line obtained after fractionated radiation treatment showed that fractionated irradiation promoted EMT (19). In this study, we examined whether single-dose irradiation can induce EMT in TE9 ESCC cells. We showed that 2 Gy of irradiation induced spindle cell-like morphologic changes, decreased expression of membranous E-cadherin, upregulated vimentin expression, and changed the localization of $\beta$-catenin from its usual membrane-bound location to the cytoplasm. Furthermore, we showed upregulation of transcription factors Slug, Snail, and Twist, which regulate EMT (32). These findings indicate that single-dose irradiation also induces EMT in ESCC cells. We also found that 2 Gy of irradiation induced EMT in other ESCC cell lines including TE10 (well differentiated type), TE11 (moderately differentiated type), and KES (well differentiated type) (data not shown).

One of the major pathways of EMT is the TGF- $\beta 1 / \mathrm{Smad}$ pathway. Components of the TGF- $\beta 1 /$ Smad signaling pathway are well known as an important signal transducers for cell proliferation, differentiation, and survival, and also serve as key factors in the regulation of cancer cell invasion and metastasis. In mice bearing tumor cells, irradiation causes increased circulating levels of TGF- $\beta 1$ as well as increased circulating tumor cells and lung metastasis (17). We similarly found that irradiation induced intracellular accumulation of TGF- $\beta 1$ and increased phosphorylation of Smad 2 and Smad3, key mediators of TGF- $\beta 1$ signaling. Although the molecular basis of a functional interaction between hypoxia, HIF-1 $\alpha$, and TGF- $\beta 1$ signaling is not well understood at this point, the simplest explanation is that TGF- $\beta 1$ levels increase in response to hypoxia (33). In this study, irradiation increased HIF-1 $\alpha$ expression at the protein level, and irradiation-induced HIF-1 $\alpha$ further upregulated the expression of MMPs. HIF-1 $\alpha$ is known as the key regulator of the cellular response to hypoxia. Induced expression of HIF-1 $\alpha$ and its target genes plays a critical role in cell growth, metastasis, and resistance to radiotherapy (33). It is noteworthy that irradiation enhanced the invasion and migration ability of TE9 cells, possibly through the upregulation of MMPs. Recent studies indicate that MMPs can activate EMT (34). MMPs cleave cell-ECM adhesion proteins and cellcell junction proteins, releasing individual epithelial cells from epithelial sheets and initiating outside-in signaling pathways that lead to widespread changes in gene transcription patterns (34). Upregulation of HIFs in cancer cells may also occur in the hypoxic intra-tumoral regions formed within primary and secondary neoplasms. Cancer cells stimulated by HIF-1 $\alpha$ show enhanced production of TGF- $\beta 1$, thus the TGF- $\beta 1 / \mathrm{Smad}$ pathway is activated and consequent production of transcription factors is increased, leading to EMT. The expression of TGF- $\beta 1$ is further enhanced, stimulating expression of cancer stem cell markers such as CD44 and CD133, which are considered to play a role in the acquisition of cancer stem cell-like properties by cancer cells. We showed that irradiation induced CD44 expression in TE9 cells. It might therefore be speculated that TE9 cells obtained stem cell-like characteristics after irradiation. It is likely that cancer stem cells are responsible for initiation, progression, recurrence, metastasis, and chemoradiotherapy resistance of cancer (35). Therefore, fractionated irradiation might induce radioresistance through the acquisition of cancer stem cell-like characteristics. In this study, we administered irradiation at a single dose of $2 \mathrm{~Gy}$, a dose that reduced TE9 cell survival to $30 \%$ in our previous study (27). Although 2 Gy of radiation killed the majority of the ESCC cells, the cells that survived after irradiation showed EMT and cancer stem cell-like characteristics with increased invasive, migration, and radioresistant phenotypes. These findings are consistent with recent reports that tumor cells can gain cancer stem cell properties as a result of EMT (36), leading to a higher probability of metastasis and radiation/drug resistance.

HDAC inhibitors are now considered to be promising anticancer agents and some of these compounds, including VPA, are near clinical stage or already on the market. Lei et al (37) showed that HDAC1 is required for TGF- $\beta 1$-induced EMT and cell migration in hepatocytes. Recently, several reports showed that HDAC inhibitor suppress EMT in various cells, including cancer cells $(31,38,39)$. HDAC inhibitors suppress metastatic potential and reverse chemoresistance in cancer cells through suppression of EMT $(30,31)$. Although the majority of reports support a suppressive effect of HDAC inhibitors on EMT, two reports show that the class I and II HDAC inhibitor VPA promotes EMT of colorectal cancer cells $(40,41)$. Therefore, we investigated the inhibitory effect of VPA on irradiation-induced EMT in ESCC cells. We found that VPA clearly inhibited EMT induced by TGF- $\beta 1$ or irradiation in these cells. Thus, VPA inhibits EMT and acquisition of cancer stem cell-like properties in ESCC cells. VPA might have a mutually exclusive effect on EMT between squamous cell carcinoma and adenocarcinoma; further studies are needed to confirm this apparent conflicting action of VPA on EMT.

We have previously demonstrated the synergistic effect of VPA on radiation therapy against ESCC (27). VPA enhances the radiosensitivity of ESCC cells through chromatin decondensation with histone hyperacetylation and increases the level of radiation-induced DNA DSBs. VPA inhibits DNA DSB repair by homologous recombination through the suppression of Rad51 and by non-homologous end joining through the acetylation of Ku70 $(27,28)$. As shown in this study, VPA can also suppress radiation-induced EMT, leading to inhibition of invasion and metastasis, and reducing resistance to further chemoradiotherapy.

We performed the same experiments using well-differentiated, moderately differentiated type, poorly differentiated types of ESCC cell and found that the poorly differentiated type showed the most remarkable changes in EMT (data not shown). Clinically, this suggests that cases of poorly differentiated squamous cell carcinoma are good candidates for combined VPA treatment with radiation therapy.

EMT is classified into three types depending on its biologic or pathologic role. Type 2 EMT is associated with inflammation and fibrosis, and is becoming increasingly recognized in adult pathologic conditions (42). Some reports show the effectiveness of chemoradiotherapy as neoadjuvant treatment for esophageal cancer. Surgery for esophageal cancer after radiotherapy is sometimes complicated by fibrosis. Concomitant usage of VPA during neoadjuvant radiotherapy might be useful to avoid fibrosis around the tumor bed. 
In conclusion, irradiation enhances the invasiveness of ESCC, partially through morphologic and molecular changes such as EMT and the induction of stem cells. Simultaneous use of the HDAC inhibitor VPA and radiotherapy might be useful not only to eradicate local disease, but also to control the systemic dissemination or metastasis associated with radiotherapy. Our data suggest that VPA might be an ideal therapeutic agent when combined with radiation for the treatment of ESCC.

\section{Acknowledgements}

This study was supported by JSPS KAKENHI (grant no. 25461909).

\section{References}

1. Jemal A, Bray F, Center MM, Ferlay J, Ward E and Forman D: Global cancer statistics. CA Cancer J Clin 61: 69-90, 2011.

2. Tachimori Y, Ozawa S, Numasaki H, Fujishiro M, Matsubara H, Oyama T, Shinoda M, Toh Y, Udagawa $\mathrm{H}$ and Uno $\mathrm{T}$ : Comprehensive registry of esophageal cancer in Japan, 2007. Esophagus 12: 101-129, 2015.

3. Osugi $\mathrm{H}$, Takemura M, Higashino M, Takada N, Lee S, Ueno M, Tanaka Y, Fukuhara K, Hashimoto Y, Fujiwara Y, et al: Causes of death and pattern of recurrence after esophagectomy and extended lymphadenectomy for squamous cell carcinoma of the thoracic esophagus. Oncol Rep 10: 81-87, 2003.

4. Ando N, Ozawa S, Kitagawa Y, Shinozawa Y and Kitajima M: Improvement in the results of surgical treatment of advanced squamous esophageal carcinoma during 15 consecutive years. Ann Surg 232: 225-232, 2000.

5. Sofia Vala I, Martins LR, Imaizumi N, Nunes RJ, Rino J, Kuonen F, Carvalho LM, Rüegg C, Grillo IM, Barata JT, et al: Low doses of ionizing radiation promote tumor growth and metastasis by enhancing angiogenesis. PLoS One 5: e11222, 2010.

6. Park JK, Jang SJ, Kang SW, Park S, Hwang SG, Kim WJ, Kang JH and Um HD: Establishment of animal model for the analysis of cancer cell metastasis during radiotherapy. Radiat Oncol 7: 153, 2012.

7. Moncharmont C, Levy A, Guy JB, Falk AT, Guilbert M, Trone JC, Alphonse G, Gilormini M, Ardail D, Toillon RA, et al: Radiation-enhanced cell migration/invasion process: A review. Crit Rev Oncol Hematol 92: 133-142, 2014.

8. Haslehurst AM, Koti M, Dharsee M, Nuin P, Evans K, Geraci J, Childs T, Chen J, Li J, Weberpals J, et al: EMT transcription factors snail and slug directly contribute to cisplatin resistance in ovarian cancer. BMC Cancer 12: 91, 2012.

9. Wintzell M, Löfstedt L, Johansson J, Pedersen AB, Fuxe J and Shoshan M: Repeated cisplatin treatment can lead to a multiresistant tumor cell population with stem cell features and sensitivity to 3-bromopyruvate. Cancer Biol Ther 13: 1454-1462, 2012.

10. Lee JM, Dedhar S, Kalluri R and Thompson EW: The epithelialmesenchymal transition: New insights in signaling, development, and disease. J Cell Biol 172: 973-981, 2006.

11. Lee MY and Shen MR: Epithelial-mesenchymal transition in cervical carcinoma. Am J Transl Res 4: 1-13, 2012.

12. Barcellos-Hoff $\mathrm{MH}$ and Akhurst RJ: Transforming growth factor-beta in breast cancer: Too much, too late. Breast Cancer Res 11: 202, 2009.

13. Morrison CD, Parvani JG and Schiemann WP: The relevance of the TGF- $\beta$ Paradox to EMT-MET programs. Cancer Lett 341: 30-40, 2013.

14. Nagaraj NS and Datta PK: Targeting the transforming growth factor-beta signaling pathway in human cancer. Expert Opin Investig Drugs 19: 77-91, 2010.

15. Wang M, Hada M, Saha J, Sridharan DM, Pluth JM and Cucinotta FA: Protons sensitize epithelial cells to mesenchymal transition. PLoS One 7: e41249, 2012.

16. Yu MA, Kiang A, Wang-Rodriguez J, Rahimy E, Haas M, Yu V, Ellies LG, Chen J, Fan JB, Brumund KT, et al: Nicotine promotes acquisition of stem cell and epithelial-to-mesenchymal properties in head and neck squamous cell carcinoma. PLoS One 7: e51967, 2012.
17. Biswas S, Guix M, Rinehart C, Dugger TC, Chytil A, Moses HL, Freeman ML and Arteaga CL: Inhibition of TGF-beta with neutralizing antibodies prevents radiation-induced acceleration of metastatic cancer progression. J Clin Invest 117: 1305-1313, 2007.

18. Tsukamoto H, Shibata K, Kajiyama H, Terauchi M, Nawa A and Kikkawa F: Irradiation-induced epithelial-mesenchymal transition (EMT) related to invasive potential in endometrial carcinoma cells. Gynecol Oncol 107: 500-504, 2007.

19. He E, Pan F, Li G and Li J: Fractionated ionizing radiation promotes epithelial-mesenchymal transition in human esophageal cancer cells through PTEN deficiency-mediated Akt activation. PLoS One 10: e0126149, 2015.

20. Ocker M: Deacetylase inhibitors - focus on non-histone targets and effects. World J Biol Chem 1: 55-61, 2010.

21. Singh TR, Shankar S and Srivastava RK: HDAC inhibitors enhance the apoptosis-inducing potential of TRAIL in breast carcinoma. Oncogene 24: 4609-4623, 2005.

22. Shankar S, Davis R, Singh KP, Kurzrock R, Ross DD and Srivastava RK: Suberoylanilide hydroxamic acid (Zolinza/ vorinostat) sensitizes TRAIL-resistant breast cancer cells orthotopically implanted in BALB/c nude mice. Mol Cancer Ther 8: 1596-1605, 2009.

23. Marks PA and Xu WS: Histone deacetylase inhibitors: Potential in cancer therapy. J Cell Biochem 107: 600-608, 2009.

24. Duenas-Gonzalez A, Candelaria M, Perez-Plascencia C, PerezCardenas E, de la Cruz-Hernandez E and Herrera LA: Valproic acid as epigenetic cancer drug: Preclinical, clinical and transcriptional effects on solid tumors. Cancer Treat Rev 34: 206-222, 2008.

25. Atmaca A, Al-Batran SE, Maurer A, Neumann A, Heinzel T, Hentsch B, Schwarz SE, Hövelmann S, Göttlicher M, Knuth A, et al: Valproic acid (VPA) in patients with refractory advanced cancer: A dose escalating phase I clinical trial. Br J Cancer 97: 177-182, 2007.

26. Mohammed TA, Holen KD, Jaskula-Sztul R, Mulkerin D, Lubner SJ, Schelman WR, Eickhoff J, Chen H and Loconte NK: A pilot phase II study of valproic acid for treatment of low-grade neuroendocrine carcinoma. Oncologist 16: 835-843, 2011.

27. Shoji M, Ninomiya I, Makino I, Kinoshita J, Nakamura K Oyama K, Nakagawara H, Fujita H, Tajima H, Takamura H, et al: Valproic acid, a histone deacetylase inhibitor, enhances radiosensitivity in esophageal squamous cell carcinoma. Int J Oncol 40: 2140-2146, 2012

28. Makita N, Ninomiya I, Tsukada T, Okamoto K, Harada S, Nakanuma S, Sakai S, Makino I, Kinoshita J, Hayashi H, et al: Inhibitory effects of valproic acid in DNA double-strand break repair after irradiation in esophageal squamous carcinoma cells. Oncol Rep 34: 1185-1192, 2015.

29. Watanabe T, Tajima H, Hironori H, Nakagawara H, Ohnishi I, Takamura H, Ninomiya I, Kitagawa H, Fushida S, Tani T, et al: Sodium valproate blocks the transforming growth factor (TGF)- $\beta 1$ autocrine loop and attenuates the TGF- $\beta 1$-induced collagen synthesis in a human hepatic stellate cell line. Int J Mol Med 28: 919-925, 2011.

30. Ruscetti M, Dadashian EL, Guo W, Quach B, Mulholland DJ, Park JW, Tran LM, Kobayashi N, Bianchi-Frias D, Xing Y, et al: HDAC inhibition impedes epithelial-mesenchymal plasticity and suppresses metastatic, castration-resistant prostate cancer. Oncogene 35: 3781-3795, 2016.

31. Sakamoto T, Kobayashi S, Yamada D, Nagano H, Tomokuni A, Tomimaru Y, Noda T, Gotoh K, Asaoka T, Wada H, et al: A Histone deacetylase inhibitor suppresses epithelial-mesenchymal transition and attenuates chemoresistance in biliary tract cancer. PLoS One 11: e0145985, 2016.

32. Thiery JP, Acloque H, Huang RY and Nieto MA: Epithelialmesenchymal transitions in development and disease. Cell 139: 871-890, 2009.

33. Paoli P, Giannoni E and Chiarugi P: Anoikis molecular pathways and its role in cancer progression. Biochim Biophys Acta 1833: 3481-3498, 2013.

34. Radisky ES and Radisky DC: Matrix metalloproteinase-induced epithelial-mesenchymal transition in breast cancer. J Mammary Gland Biol Neoplasia 15: 201-212, 2010.

35. Schatton T and Frank MH: Cancer stem cells and human malignant melanoma. Pigment Cell Melanoma Res 21: 39-55, 2008.

36. Castellanos JA, Merchant NB and Nagathihalli NS: Emerging targets in pancreatic cancer: Epithelial-mesenchymal transition and cancer stem cells. Onco Targets Ther 6: 1261-1267, 2013. 
37. Lei W, Zhang K, Pan X, Hu Y, Wang D, Yuan X, Shu G and Song J: Histone deacetylase 1 is required for transforming growth factor-beta1-induced epithelial-mesenchymal transition. Int J Biochem Cell Biol 42: 1489-1497, 2010.

38. Bruzzese F, Leone A, Rocco M, Carbone C, Piro G, Caraglia M, Di Gennaro E and Budillon A: HDAC inhibitor vorinostat enhances the antitumor effect of gefitinib in squamous cell carcinoma of head and neck by modulating ErbB receptor expression and reverting EMT. J Cell Physiol 226: 2378-2390, 2011.

39. Mateen S, Raina K, Agarwal C, Chan D and Agarwal R: Silibinin synergizes with histone deacetylase and DNA methyltransferase inhibitors in upregulating E-cadherin expression together with inhibition of migration and invasion of human non-small cell lung cancer cells. J Pharmacol Exp Ther 345: 206-214, 2013.
40. Feng J, Cen J, Li J, Zhao R, Zhu C, Wang Z, Xie J and Tang W: Histone deacetylase inhibitor valproic acid (VPA) promotes the epithelial mesenchymal transition of colorectal cancer cells via up regulation of Snail. Cell Adhes Migr 9: 495-501, 2015.

41. Ji M, Lee EJ, Kim KB, Kim Y, Sung R, Lee SJ, Kim DS and Park SM: HDAC inhibitors induce epithelial-mesenchymal transition in colon carcinoma cells. Oncol Rep 33: 2299-2308, 2015.

42. Kovacic JC, Mercader N, Torres M, Boehm M and Fuster V: Epithelial-to-mesenchymal and endothelial-to-mesenchymal transition: From cardiovascular development to disease. Circulation 125: 1795-1808, 2012. 